\title{
Puerarin Alleviates Neuropathic Pain by Inhibiting Neuroinflammation in Spinal Cord
}

\author{
Ming Liu, ${ }^{1,2,3}$ Kaijun Liao, ${ }^{3}$ Changxi Yu, ${ }^{3}$ Xuejun Li, ${ }^{1,4}$ Suhuan Liu, ${ }^{1,5}$ and Shuyu Yang ${ }^{1,4}$ \\ ${ }^{1}$ Xiamen Diabetes Institute, The First Affiliated Hospital of Xiamen University, 55 Zhenhai Road, Xiamen 361003, China \\ ${ }^{2}$ Huazhong University of Science \& Technology, China \\ ${ }^{3}$ Department of Pharmacology, College of Pharmacy, Fujian Medical University, China \\ ${ }^{4}$ Division of Endocrinology and Diabetes, The First Affiliated Hospital of Xiamen University, China \\ ${ }^{5}$ Central Laboratory, The First Affiliated Hospital of Xiamen University, China
}

Correspondence should be addressed to Suhuan Liu; liusuhuan@gmail.com and Shuyu Yang; xmyangshuyu@126.com

Received 17 February 2014; Revised 9 June 2014; Accepted 10 June 2014; Published 24 June 2014

Academic Editor: Bing Du

Copyright (C) 2014 Ming Liu et al. This is an open access article distributed under the Creative Commons Attribution License, which permits unrestricted use, distribution, and reproduction in any medium, provided the original work is properly cited.

\begin{abstract}
Neuropathic pain responds poorly to drug treatments, and partial relief is achieved in only about half of the patients. Puerarin, the main constituent of Puerariae Lobatae Radix, has been used extensively in China to treat hypertension and tumor. The current study examined the effects of puerarin on neuropathic pain using two most commonly used animal models: chronic constriction injury (CCI) and diabetic neuropathy. We found that consecutive intrathecal administration of puerarin (4-100 nM) for 7 days inhibited the mechanical and thermal nociceptive response induced by CCI and diabetes without interfering with the normal pain response. Meanwhile, in both models puerarin inhibited the activation of microglia and astroglia in the spinal dorsal horn. Puerarin also reduced the upregulated levels of nuclear factor- $\kappa \mathrm{B}(\mathrm{NF}-\kappa \mathrm{B})$ and other proinflammatory cytokines, such as IL- 6 , IL- $1 \beta$, and TNF$\alpha$, in the spinal cord. In summary, puerarin alleviated CCI- and diabetes-induced neuropathic pain, and its effectiveness might be due to the inhibition of neuroinflammation in the spinal cord. The anti-inflammation effect of puerarin might be related to the suppression of spinal NF- $\kappa \mathrm{B}$ activation and/or cytokines upregulation. We conclude that puerarin has a significant effect on alleviating neuropathic pain and thus may serve as a therapeutic approach for neuropathic pain.
\end{abstract}

\section{Introduction}

Neuropathic pain is caused by aberrant sensory processing in either the peripheral- and/or the central nervous system $(\mathrm{CNS})$ and can be induced by a variety of factors, including traumatic damage, infection, and diabetes [1]. Abnormal neuronal activity plays a fundamental role in the pathogenesis of neuropathic pain, while current treatments that suppress aberrant neuronal activity generally lack efficacy, in addition to their many undesirable side effects [2]. Recent studies have indicated that immune response of the CNS, in which glial cells are critically involved, plays an important role in the development and persistence of neuropathic pain $[3,4]$. Hyperalgesia and allodynia are frequently induced by increased levels of proinflammatory cytokines, including TNF- $\alpha$, IL- $1 \beta$, and IL- 6 , in the CNS, and the resulting or accompanying activation of glial cells [5]. Thus cytokines and glial cells in the CNS have been recognized as powerful modulators of nociception and hold potent potential to the control of neuropathic pain [6].

Puerarin, the main constituent of Radix Puerariae, has a variety of pharmacology characteristics [7]. Puerarin has been shown to effectively inhibit proinflammatory cytokine production and/or glia cell activation in a variety of diseases [8-10]. We speculated that puerarin could also be effective in the intrathecal treatment of neuropathic pain via inhibition of spinal inflammation; thus the present study was designed to investigate the effects of puerarin on neuropathic pain using two most commonly used rodent pain models (partial sciatic nerve injury and diabetes). In addition to pain, the effects of puerarin on proinflammatory cytokine production and glial activation in the spinal cord were also examined. 


\section{Materials and Methods}

2.1. Animals. Male Sprague-Dawley rats (220-250 g) were purchased from Shanghai Experimental Animal Center, Chinese Academy of Sciences (Shanghai, China). The rats were housed in a temperature-controlled room $\left(22-25^{\circ} \mathrm{C}\right)$ in plastic cages ( 5 animals per cage) with a 12-hour light/dark cycle and had free access to food and water. All animal experiments were approved by Xiamen University Animal Care and Use Committee. All efforts were made to minimize the animal suffering and the number of animals used.

2.2. Drugs. For intrathecal injections, Puerarin and fluorocitrate were dissolved in artificial cerebrospinal fluid (ACSF). The solution was completed in distilled water. Puerarin and fluorocitrate and all other reagents were purchased from Sigma-Aldrich.

2.3. Intrathecal Catheterization. For intrathecal drug administration, intrathecal catheterization [11] was performed by advancing the PE-5 catheter $8.5 \mathrm{~cm}$ caudally to the lumbar enlargement through an incision in the cisternal membrane of rats that were under isoflurane mask anesthesia. The catheter was externalized and secured to the musculature at the incision site. Rats showing any neurologic dysfunction, such as paralysis or urine incontinence after catheterization, were euthanized. A proper location of the catheter was confirmed by the hind limb paralysis after $10 \mu \mathrm{L}$ intrathecal injection of $2 \%$ lidocaine (Sigma) 1 day after catheterization. Only rats displaying normal grooming, ambulation, and weight gain after catheterization were used in the following study.

2.4. Type 1 Diabetic Neuropathic Pain Model. Diabetes was induced in rats by a single intraperitoneal (i.p.) injection of streptozotocin (STZ, Sigma; $60 \mathrm{mg} / \mathrm{kg}$, i.p.) [12]. Agematched control rats received an equal volume of vehicle ( $0.01 \mathrm{M}$ citrate buffer, $\mathrm{pH} 4.5)$. Blood was collected via the tail vein at $72 \mathrm{~h}$ after STZ injection to measure glucose concentration. Rats with a blood glucose level over $16.67 \mathrm{mmol} / \mathrm{L}$ were considered to be diabetic and included in the subsequent experiments. Puerarin (4, 20, and $100 \mathrm{nM}$ ), fluorocitrate $(1 \mathrm{nM}$, served as a positive control), or vehicle was administered intrathecally once daily for 7 consecutive days beginning on day 20 after STZ injection. The mechanical withdrawal threshold of the right hind paw was measured before STZ injection (baseline), before drug treatment (predose), and $60 \mathrm{~min}$ after each drug administration (postdose) in the morning.

2.5. Chronic Constriction Injury (CCI) Model of Neuropathic Pain. The rat CCI model of neuropathic pain was produced according to the method described before [13]. Adult male Sprague-Dawley rats were anaesthetized with chloral hydrate (400 mg/kg, i.p.). The right common sciatic nerve was isolated at mid-thigh level and loosely ligated using chromic gut suture (5-0) at four sites separated by an interval of $1 \mathrm{~mm}$. For sham surgery, the right sciatic nerve was exposed, but the nerve was not ligated. All animals were allowed 3 days to recover from the surgery. Puerarin $(4,20$, and $100 \mathrm{nM})$, fluorocitrate $(1 \mathrm{nM})$, or vehicle was administered intrathecally once daily for 7 consecutive days beginning on day 4 postoperatively. The mechanical withdrawal threshold was measured before surgery (baseline), before drug treatment (predose), and 60 min after each drug administration (postdose) in the morning.

2.6. Mechanical Allodynia. Mechanical allodynia was measured using a commercially available electronic von Frey apparatus (Model 2390; IITC Life Science Inc., Woodland Hills, CA) as described previously [14] with minor modifications. Rats were placed into a Plexiglas box on a steel mesh floor. Pressure was applied to the center of the hind paw with the von Frey filament in an upward motion until foot withdrawal. The withdrawal threshold was automatically recorded. The maximum strength of the filament was $55 \mathrm{~g}$. The procedure was repeated after approximately $10 \mathrm{~min}$ for each daily session, and the average mechanical withdrawal threshold (MWT) was calculated.

2.7. Measurements of NF- $\kappa B$ DNA Binding Activity and Inflammatory Cytokines. NF- $\kappa \mathrm{B}$ DNA binding activity measurements were performed using a commercially supplied $\mathrm{NF}-\kappa \mathrm{B}$ transcription factor binding assay kit (Cayman Chemical, CA, USA) according to the manufacturer's suggested protocol. The assay is based on the principle that only the active form of NF- $\kappa \mathrm{B}$ in the sample binds to oligonucleotide containing the NF- $\kappa \mathrm{B}$ consensus site $\left(5^{\prime}\right.$-GGGACTTTCC- $\left.3^{\prime}\right)$ that is immobilized on the microtiter plate [15]. Briefly, lumbar section of the spinal cord was homogenized in lysis buffer. The nuclear extract was prepared using a nuclear extract kit (Cayman Chemical), and samples of nuclear extract were first incubated overnight at $4^{\circ} \mathrm{C}$ in wells precoated with a dsDNA sequence corresponding to the NF- $\kappa$ B consensus motif. The NF- $\kappa$ B consensus motif of the assay should bind both human and rat p65. After 5 washes, the samples were incubated overnight at $4^{\circ} \mathrm{C}$ with primary antibody (rabbit polyclonal, Cayman Chemical) to the p65 subunit of NF- $\kappa \mathrm{B}$. The primary antibody against the $\mathrm{p} 65$ subunit of NF- $\kappa \mathrm{B}$ used in the assay system is accessible only when NF- $\kappa \mathrm{B}$ is activated and bound to its target DNA. Subsequently, samples were incubated for 60 minutes with an HRP-conjugated goat antirabbit secondary antibody (Cayman Chemical), followed by colorimetric detection at $450 \mathrm{~nm}$ (Multiskan FC, Thermo Scientific). After background subtraction, absorbance measures were referred to a standard curve obtained from a series of duplicate wells containing measured amounts of human recombinant p65 (Cayman Chemical) and then converted to an estimate of the quantity of p65/well, which was normalized by dividing the p65 estimate by the total amount of protein measured in the sample. TNF- $\alpha$, IL-6, and IL- $1 \beta$ were quantified using ELISA kits (Abcam, USA) according to the manufacturer's instructions.

2.8. Immunohistochemistry. Rats were anesthetized with sodium pentobarbital (50 mg/kg, i.p.), perfused intracardially 
with $300 \mathrm{~mL}$ of $0.9 \%$ saline followed by $300 \mathrm{~mL}$ of $4 \%$ paraformaldehyde in $0.1 \mathrm{M}$ phosphate buffer ( $\mathrm{PB}, \mathrm{pH} 7.2-$ $7.4,4^{\circ} \mathrm{C}$ ). Lumbar spinal segments were removed, postfixed overnight at $4^{\circ} \mathrm{C}$, and kept in $30 \%$ sucrose in $0.1 \mathrm{M}$ phosphatebuffered saline (PBS) at $4^{\circ} \mathrm{C}$. Dissected tissue was mounted in OCT compound and frozen at $-20^{\circ} \mathrm{C}$. Transverse spinal cord sections $(10 \mu \mathrm{m})$ were prepared using a cryostat (Microm HM550) and placed in PBS. Sections were washed in $0.01 \mathrm{M}$ PBS twice for $10 \mathrm{~min}$ and blocked for $1 \mathrm{~h}$ in $5 \%$ bovine serum albumin and $0.1 \%$ Triton X-100. Free floating tissue sections were incubated overnight at $4^{\circ} \mathrm{C}$ on a rocker with a rabbit polyclonal antiastroglia marker-GFAP antibody (Abcam; 1:1000) or a rabbit polyclonal antimicroglia marker-Iba-1 antibody (Abcam; $1: 1000$ ). Tissue samples were then washed twice with PBS for $8 \mathrm{~min}$ each and incubated with FITC- or Cy3-conjugated anti-rabbit antibody $(1: 300$, Jackson Immuno Research Laboratories Inc.) in blocking solution without Triton X-100 for $1 \mathrm{~h}$ at room temperature in the dark. Control staining was performed by omitting the primary antibody. Fluorescent images were captured with a digital camera (Olympus). The percentage of positive immunostained area in the dorsal horn was analyzed using Image-Pro software (Plus Version 6).

2.9. Western Blot. Nuclear and cytoplasmic protein was extracted with a nuclear and cytoplasmic extract kit (Cayman Chemical). Protein content of each sample was determined using the BioRad protein assay (BioRad Laboratories) according to manufacturer's protocol. For immunoblot analysis, $25 \mu \mathrm{g}$ of protein was subjected to SDS-PAGE, transferred to a membrane, and probed with specific antibodies: NF$\kappa \mathrm{B}$ p65 (1:5000, Abcam); phospho-NF- $\kappa$ B p65 (Ser 536, $1: 5000$, Abcam); Lamin B (1:2000, Abcam); $\beta$-actin (1:2000, Abcam). Secondary antibody HRP-conjugated secondary antibodies (Santa Cruz Biotechnology, 1:5000) were used to detect binding of antibodies. The membrane was incubated with Clarity Western ECL Substrate (Bio-Rad Laboratories, CA, USA), and the target proteins were then visualized and quantitated using a LAS-3000 luminescent image analyzer (Fujifilm). The results were expressed as a relative ratio of the target protein to reference protein.

2.10. Statistical Analysis. The antimechanical allodynia effects were evaluated by the increment of the MWT after drugs treatment and expressed as percentage of maximal possible effect (\%MPE): MPE\% = [(postdose threshold $)-$ (predose threshold)]/[(baseline threshold) - (predose threshold)] $\times 100$ [16]. It is possible to obtain a negative value of \%MPE if the MWT was decreased after treatments, that is, to enhance the allodynic response. Data from the behavior test, ELISA, and immunohistochemistry were analyzed using two-way ANOVA or one-way ANOVA followed by the LSD $t$-test for post hoc analysis. All of the data are presented as mean \pm SEM, and all statistical analyses were performed using SPSS software version 16.0 (SPSS Inc., Chicago, IL, USA). A $P$ value of $<0.05$ was considered statistically significant.

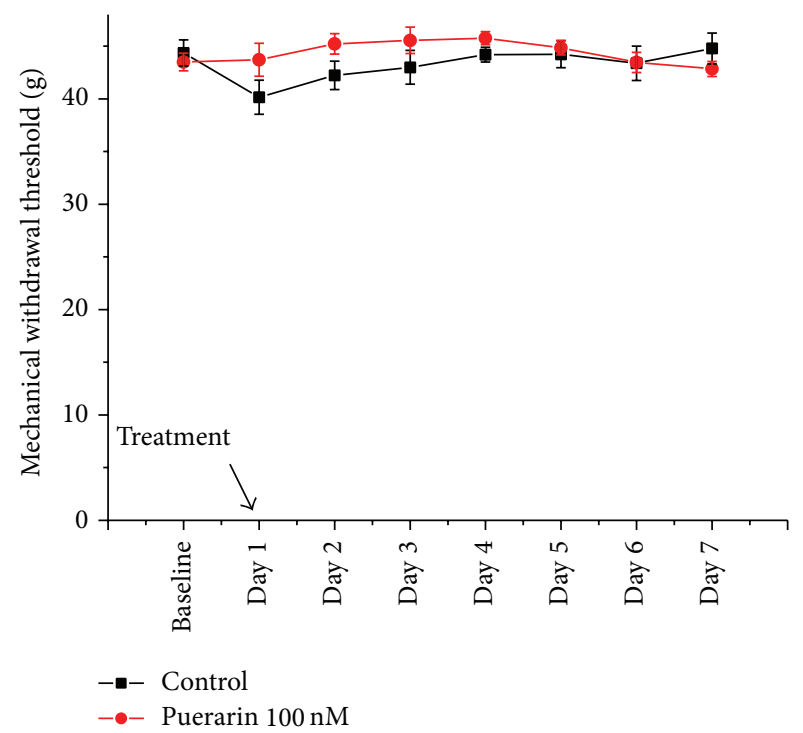

FIgURE 1: The effects of puerarin on mechanical stimulus test in normal rats. Normal rats received intrathecal $100 \mathrm{nmol}$ puerarin or vehicle. The mechanical withdrawal thresholds (g) of the right hind paws were measured $60 \mathrm{~min}$ after drug administration. Data were presented as mean \pm SEM. Each group consisted of 5 rats. At $100 \mathrm{nM}$, puerarin did not affect mechanical withdrawal threshold.

\section{Results}

3.1. Effects of Puerarin on Pain in Normal Rats. Puerarin (100 nM, i.t., for 7 consecutive days) did not affect MWT (Figure 1).

3.2. Effects of Puerarin on CCI- and Diabetes-Induced Neuropathic Pain. Post hoc tests showed CCI and diabetes significantly decreased $(P<0.001$, versus sham control) MWT to mechanical stimulation (Figures 2(a) and 2(c), $P<0.001$, versus sham control), demonstrating the development of mechanical allodynia which persisted for the entire observation period. Comparable to fluorocitrate, puerarin significantly reduced mechanical allodynia in both CCIand diabetes models compared to the vehicle (Figures 2(a)2(d)). The MPE of puerarin for CCI- and diabetes-induced neuropathic pain on day 7 was $16.41 \% \pm 5.66 \%$ at $4 \mathrm{nM}$, $43.41 \% \pm 2.75 \%$ at $20 \mathrm{nM}$, and $64.2 \% \pm 3.71 \%$ at $100 \mathrm{nM}$ for CCI and $23.93 \% \pm 5.49 \%$ at $4 \mathrm{nM}, 49.11 \% \pm 4.23 \%$ at $20 \mathrm{nM}$, and $62.07 \% \pm 6.34 \%$ at $100 \mathrm{nM}$ for diabetes, respectively (Figures 2(b) and 2(d)). The MPE of fluorocitrate was 69.9\% \pm $5.48 \%$ for CCI and $76.24 \% \pm 5.27 \%$ for diabetes, respectively (Figures 2(b) and 2(d)).

\subsection{Spinal Microglia and Astroglia Activation in CCI and} Diabetic Rats. In CCI groups, staining of the microglia activation marker Iba-1 was barely detectable in the spinal cord in sham control rats (Figure 3(a)). The number of Iba1 immunoreactive cells significantly increased in the dorsal horn ipsilateral to the CCI injury on day 11 after surgery (Figures 3(a) and 3(c), $P<0.001$ ). Puerarin reduced the enhanced Iba-1 immunoreactivity in the spinal cord (Figures 


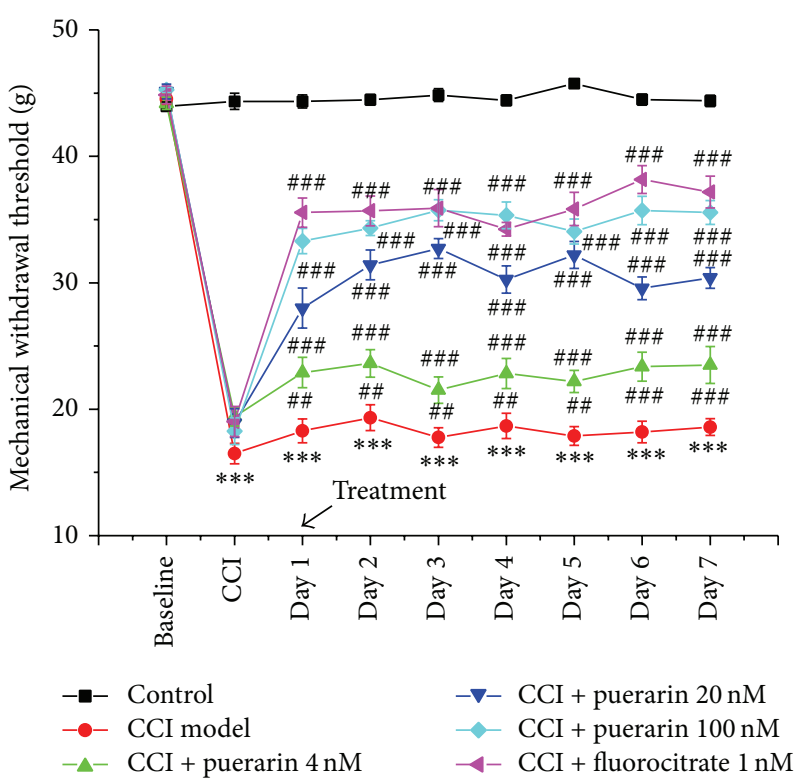

(a)

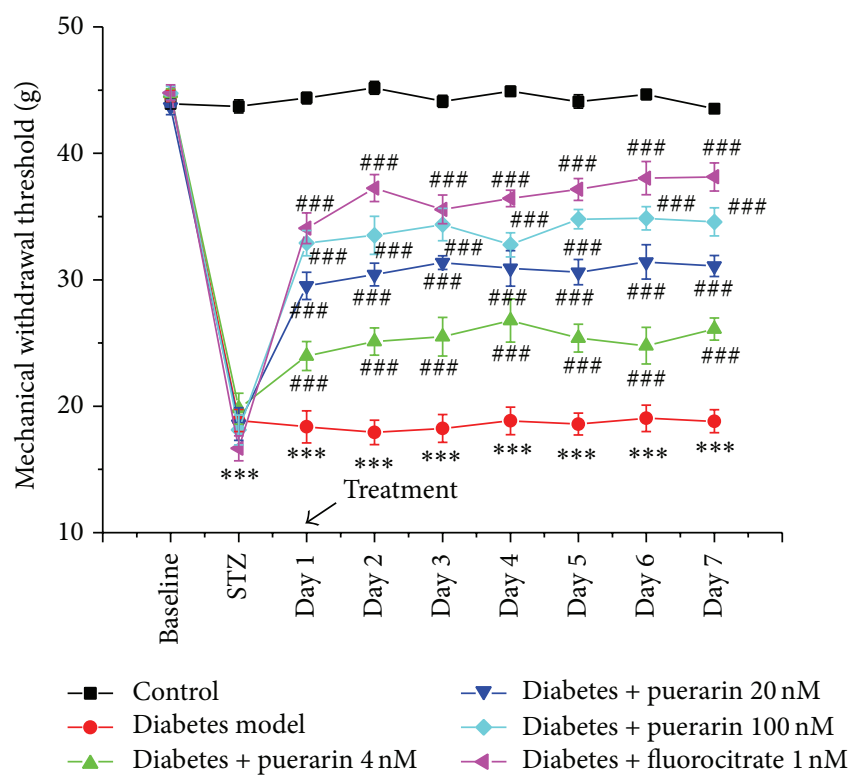

(c)

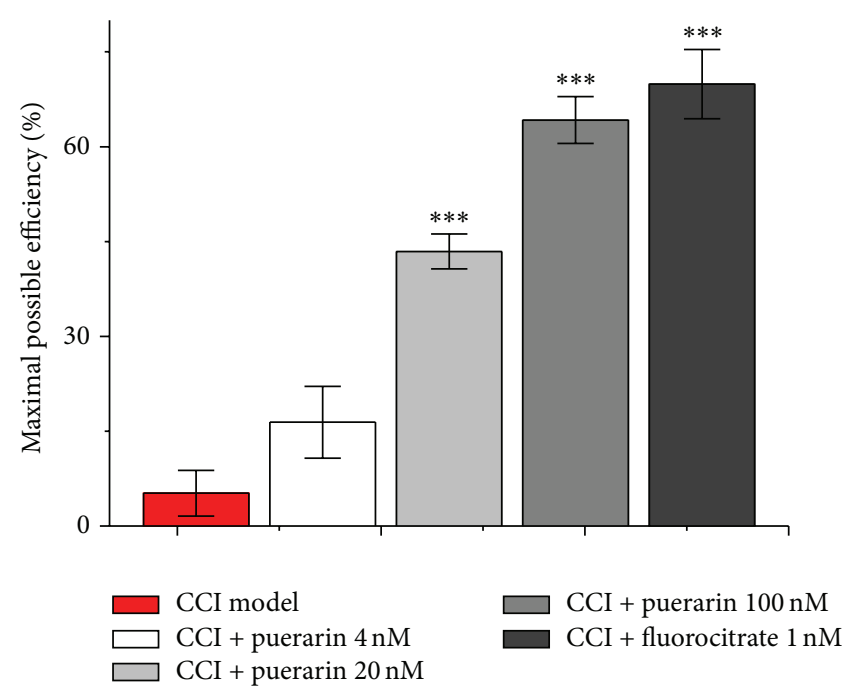

(b)

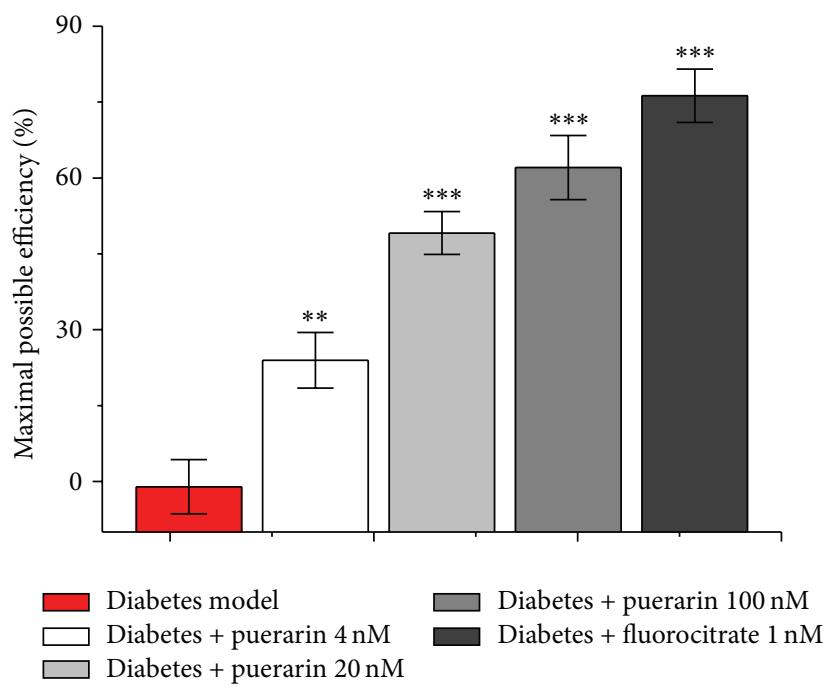

(d)

Figure 2: The effects of puerarin on CCI- and diabetes-induced mechanical allodynia. (a), (c) The temporal profile of mechanical withdrawal threshold on CCI- (a) and diabetes- (c) induced mechanical allodynia; (b), (d) the maximal possible efficiency of puerarin on the 11th day after CCI surgery (b) and the 27th day after streptozotocin injection; CCI: chronic constriction injury. Data are presented as mean \pm SEM. ${ }^{* * *} P<0.001$, model group versus control group; ${ }^{\#} P<0.05,{ }^{\# \#} P<0.01$, ${ }^{\# \# \#} P<0.001$, puerarin treatment group versus model group (one-way ANOVA with post hoc LSD $t$-test). Each group consisted of 6-10 rats.

3 (a) and 3(c), $P<0.001$ for puerarin $20,100 \mathrm{nM})$. Rats receiving sham CCI surgery showed a low-level staining for GFAP, an astroglia activation marker, in the spinal cord (Figure 3(b)). The number of GFAP immunoreactive cells significantly increased in the dorsal horn ipsilateral to CCI injury on day 11 after surgery (Figures 3(b) and 3(c), $P<$ $0.001)$. Puerarin reduced the increase of GFAP immunoreactivity in the spinal cord caused by CCI (Figures 3(b) and 3 (c), $P<0.001$ for puerarin $20,100 \mathrm{nM}$ ).
As for the diabetic groups, there is a similar trend. A low level and diffuse staining of Iba-1 was noticed in the spinal cord of rats receiving vehicle injection (Figure 4(a)). The number of Iba-1 immunoreactive cells dramatically increased in the dorsal horn on day 27 after diabetes induction (Figures 4(a) and 4(c), $P<0.001)$. Puerarin attenuated the increase of Iba-1 immunoreactivity in the spinal cord in the diabetic rats (Figures 4(a) and 4(c), $P<0.001$ for puerarin 20, $100 \mathrm{nM}$ ). Staining of GFAP in the spinal cord 


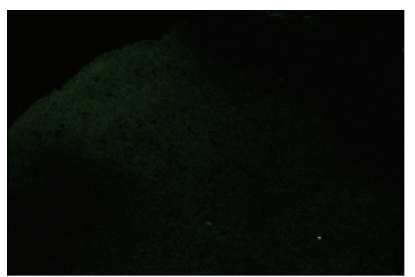

Control

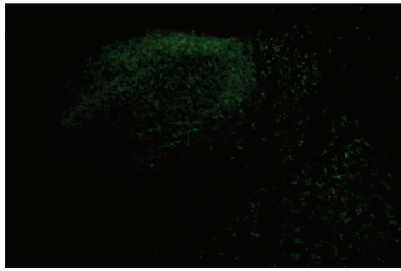

Puerarin $20 \mathrm{nM}$

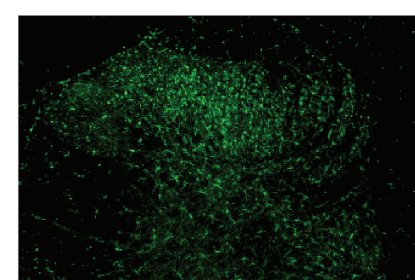

Chronic constriction injury

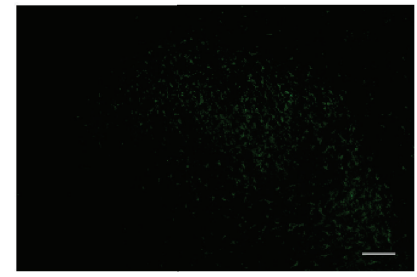

Puerarin $100 \mathrm{nM}$

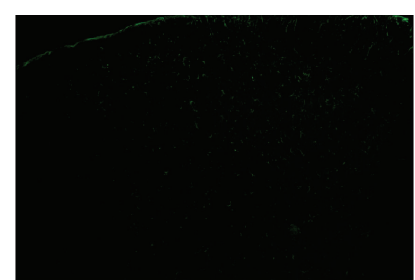

Control

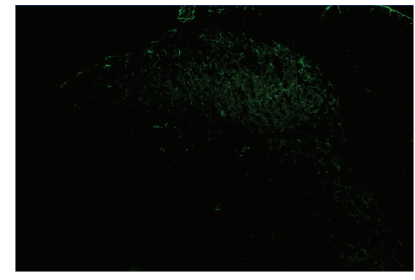

Puerarin $20 \mathrm{nM}$

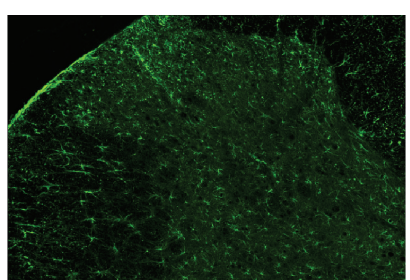

Chronic constriction injury

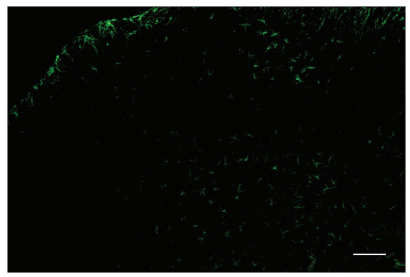

Puerarin $100 \mathrm{nM}$

(a)

(b)

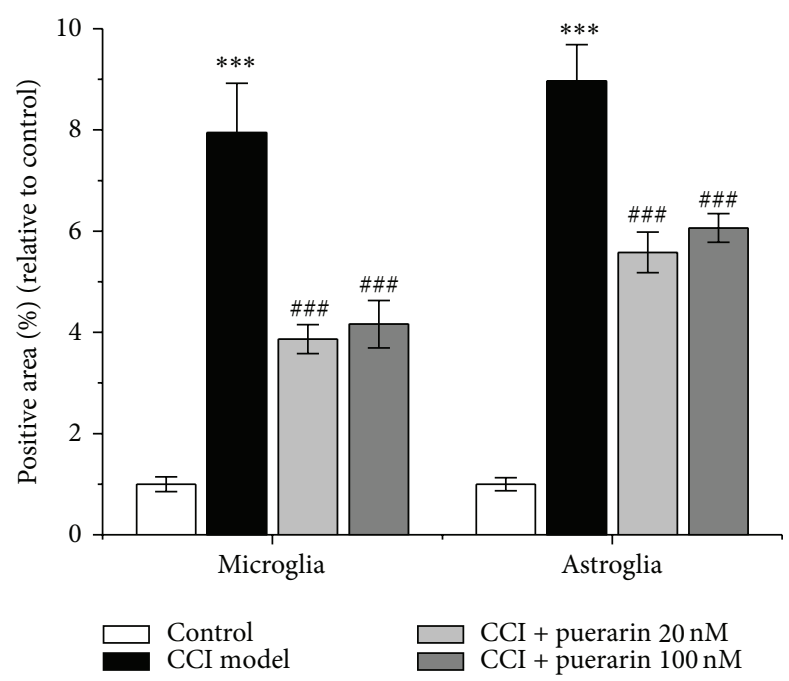

(c)

FIgURE 3: The effects of puerarin on CCI-induced microglia and astroglia activation in ipsilateral spinal dorsal horn of rats. (a) and (b) Representative images of ipsilateral spinal dorsal microglia (a) and astroglia (b) activation in control rats, CCI rats, CCI rats receiving vehicle, 20 and $100 \mathrm{nM}$ puerarin on the 11th day after CCI surgery. Scale bar: $100 \mu \mathrm{m}$. (c) Quantification of microglia activation in the spinal cord on the 11th day after CCI surgery. CCI: chronic constriction injury. Data are presented as mean \pm SEM. ${ }^{* * *} P<0.001$, CCI model versus control; ${ }^{\# \# \#} P<0.001$, puerarin versus CCI model (one-way ANOVA with post hoc LSD $t$-test). Each group consisted of 6-10 rats.

was barely noticeable in rats receiving vehicle (Figure $4(\mathrm{~b})$ ), while significantly increased in the dorsal horn on day 27 after diabetes induction (Figures 4(b) and 4(c), $P<0.001$ ). Puerarin reversed the elevated GFAP immunoreactivity in the spinal cord (Figures 4(b) and 4(c), $P<0.001$ for puerarin 20, $100 \mathrm{nM})$.

3.4. Effect of Puerarin on TNF- $\alpha, I L-1 \beta$, and IL-6 Production and NF- $\kappa B$ Activation in CCI and Diabetic Rats. CCI increased TNF- $\alpha$, IL- $1 \beta$, and IL- 6 production and NF- $\kappa \mathrm{B}$ activation in the spinal cord $(P<0.001$ versus control, Figure 5). Puerarin attenuated CCI-induced increase of TNF$\alpha$, IL-1 $\beta$, and IL-6 $(P<0.001$ for IL-1 $\beta$ and IL-6 at 20, $100 \mathrm{nM}, P<0.01$ and $P<0.001$ for TNF- $\alpha$ at $20 \mathrm{nM}$ and $100 \mathrm{nM}$, resp., Figure 5(a)). The elevated NF- $\kappa \mathrm{B}$ DNA binding activities were also significantly reduced with puerarin treatment $(P<0.001$ and $P<0.01$ at $20 \mathrm{nM}$ and $100 \mathrm{nM}$, resp., Figure 5(b)). Moreover, the overexpression of $\mathrm{NF}-\kappa \mathrm{B}$ p 65 and p 65 nucleus translocation was significantly reduced with puerarin treatment $(P<0.01$, Figures $5(\mathrm{c})$ and $5(d))$.

With the same pattern, TNF- $\alpha$, IL- $1 \beta$, and IL- 6 production and $\mathrm{NF}-\kappa \mathrm{B}$ activation were significantly elevated in the spinal cord of diabetic rats $(P<0.001$ versus control, Figure 5). Puerarin at 20 and $100 \mathrm{nM}$ decreased the diabetes-induced elevation of TNF- $\alpha$, IL- $1 \beta$, and IL- 6 $(P<0.001$, Figure 5(a)) and NF- $\kappa$ B DNA binding activities $(P<0.01$ and $P<0.001$ at $20 \mathrm{nM}$ and $100 \mathrm{nM}$, resp., Figure $5(\mathrm{~b}))$. Besides, the overexpression of NF- $\kappa \mathrm{B}$ p 65 and p65 nucleus translocation was significantly inhibited with puerarin treatment $(P<0.01$, Figures 5(c) and $5(d))$. 


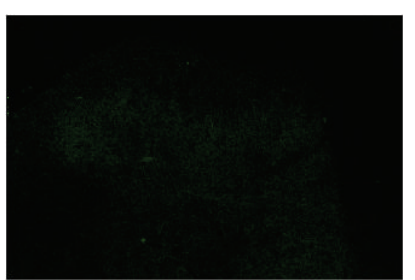

Control

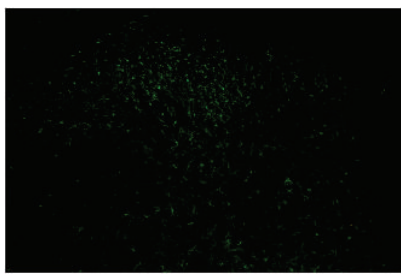

Puerarin $20 \mathrm{nM}$

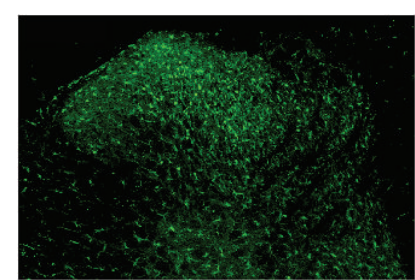

Diabetes

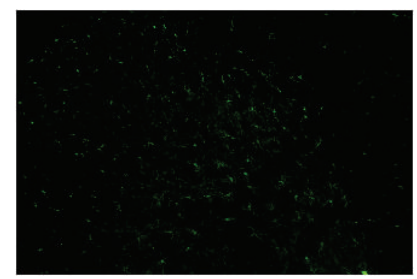

Puerarin $100 \mathrm{nM}$

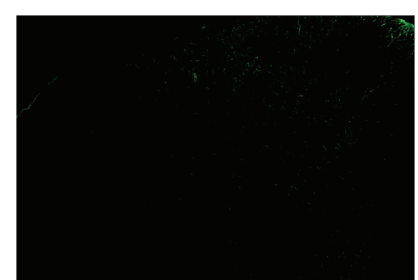

Control

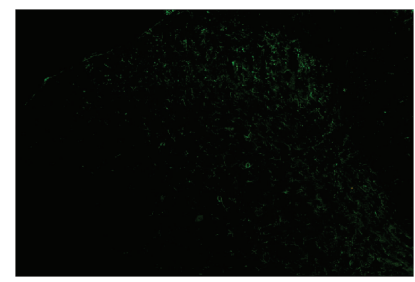

Puerarin $20 \mathrm{nM}$

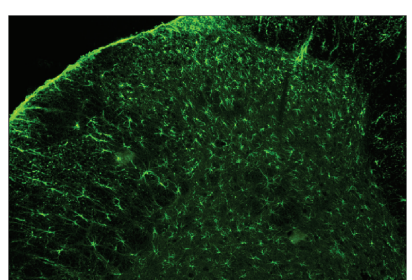

Diabetes

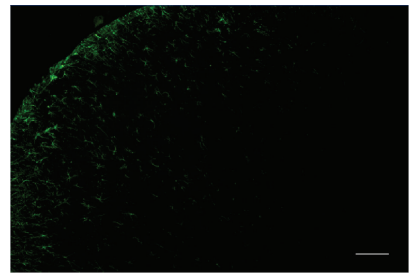

Puerarin $100 \mathrm{nM}$

(a)

(b)

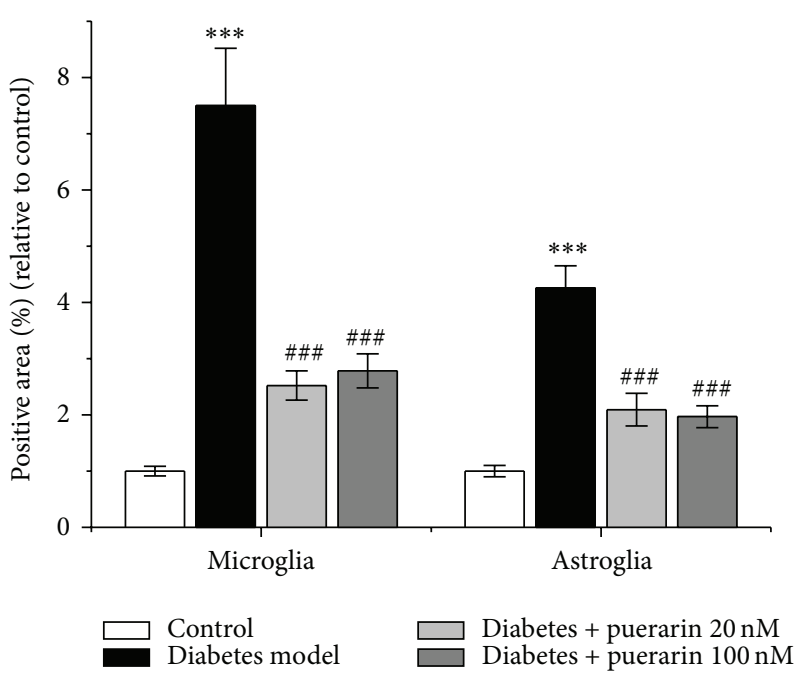

(c)

FIGURE 4: The effects of puerarin on diabetes-induced microglia and astroglia activation in ipsilateral spinal dorsal horn of rats. (a) and (b) Representative images of ipsilateral spinal dorsal microglia (a) and astroglia (b) activation in control rats, diabetic rats receiving vehicle, 20 and $100 \mathrm{nM}$ puerarin on the 27th day after streptozotocin injection. Scale bar: $100 \mu \mathrm{m}$. (c) Quantification of microglia and astroglia activation in the spinal cord on the 27 th day after streptozotocin injection. Data are presented as mean \pm SEM. ${ }^{* * *} P<0.001$, diabetic rats versus control; ${ }^{\# \# \#} P<0.001$, puerarin versus diabetes model (one-way ANOVA with post hoc LSD $t$-test). Each group consisted of 6-10 rats.

\section{Discussion}

Neuropathic pain is caused by aberrant sensory processing in either the peripheral nervous system and/or the central nervous system and is currently lacking efficacious therapy. We found that consecutive intrathecal administration of puerarin to rats inhibited the mechanical and thermal nociceptive response induced by CCI and diabetes, which might acted through inhibiting the activation of microglia and astroglia in the spinal dorsal horn and reducing the upregulated levels of nuclear factor $-\kappa \mathrm{B}(\mathrm{NF}-\kappa \mathrm{B})$ and proinflammatory cytokines including IL-6, IL- $1 \beta$, and TNF- $\alpha$, in the spinal cord.

Neuropathic pain is characterized by spontaneous pain, increased responsiveness to pain stimuli (hyperalgesia), and pain perceived in response to normally nonnoxious stimuli (allodynia) [1]. A growing body of literature indicates that the enhanced spinal neuroimmune and neuroinflammatory activities initiate and maintain neuropathic pain after the primary nerve injury $[6,17]$. Specifically, the proinflammatory cytokines such as TNF- $\alpha$, IL- $1 \beta$, and IL- 6 have been strongly implicated in the initiation and development of neuropathic pain after nerve injury $[18,19]$. Neuropathic pain is usually accompanied by peripheral and central nervous system damage. Nerve injury leads to a rapid release of pain-related mediators, such as TNF- $\alpha$, IL- $1 \beta$, IL- 6 , and prostaglandins, resulting in inflammatory responses that sensitize the CNS and facilitate pain processing [20]. NF- $\kappa$ B plays a vital role in these processes and is an important mediator in the regulation of proinflammatory cytokines and inflammatory and immune responses [21]. A number of inflammatory mediators (i.e., TNF- $\alpha$, IL- $1 \beta$, IL-6, NO, and TGF-1 $\beta$ ) that are implicated in the modulation of the neuropathic pain 


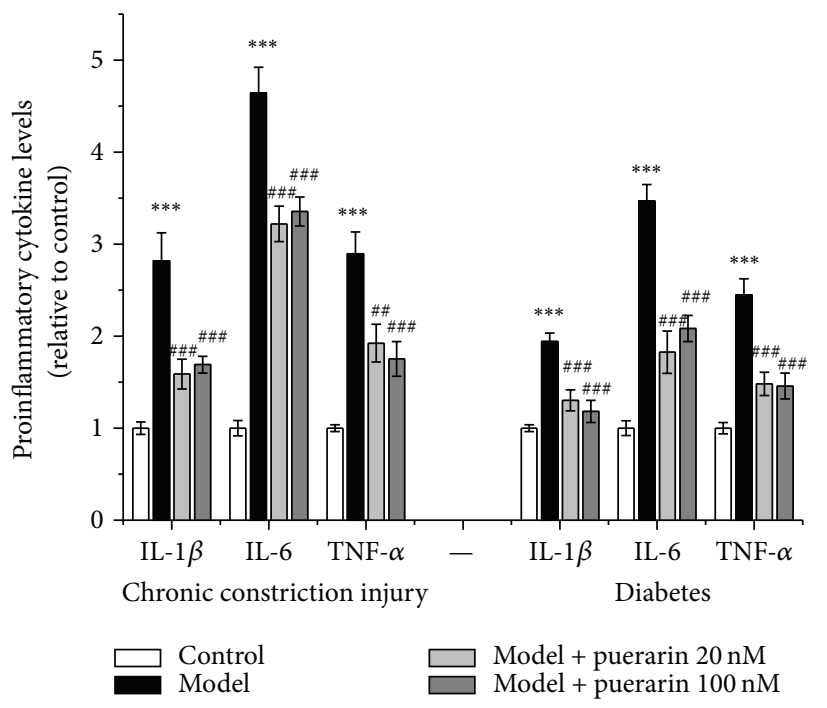

(a)

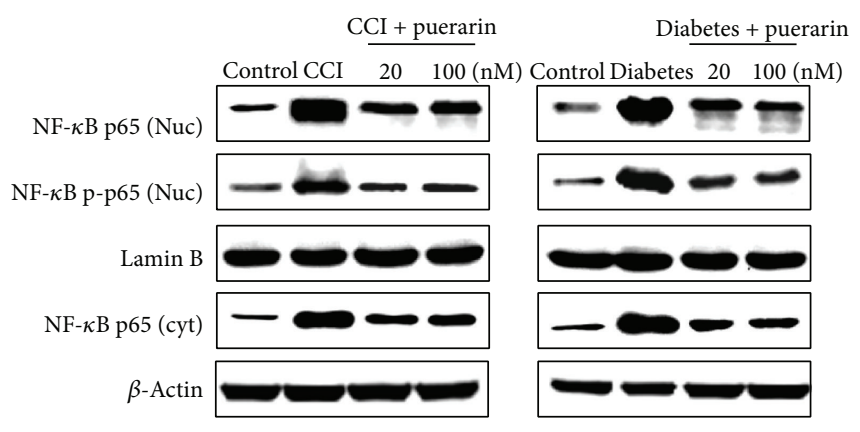

(c)

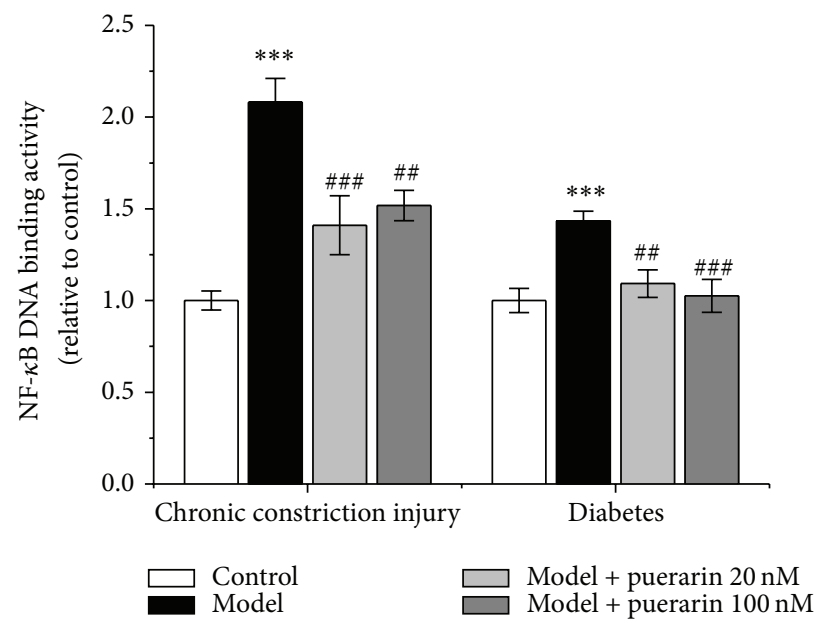

(b)

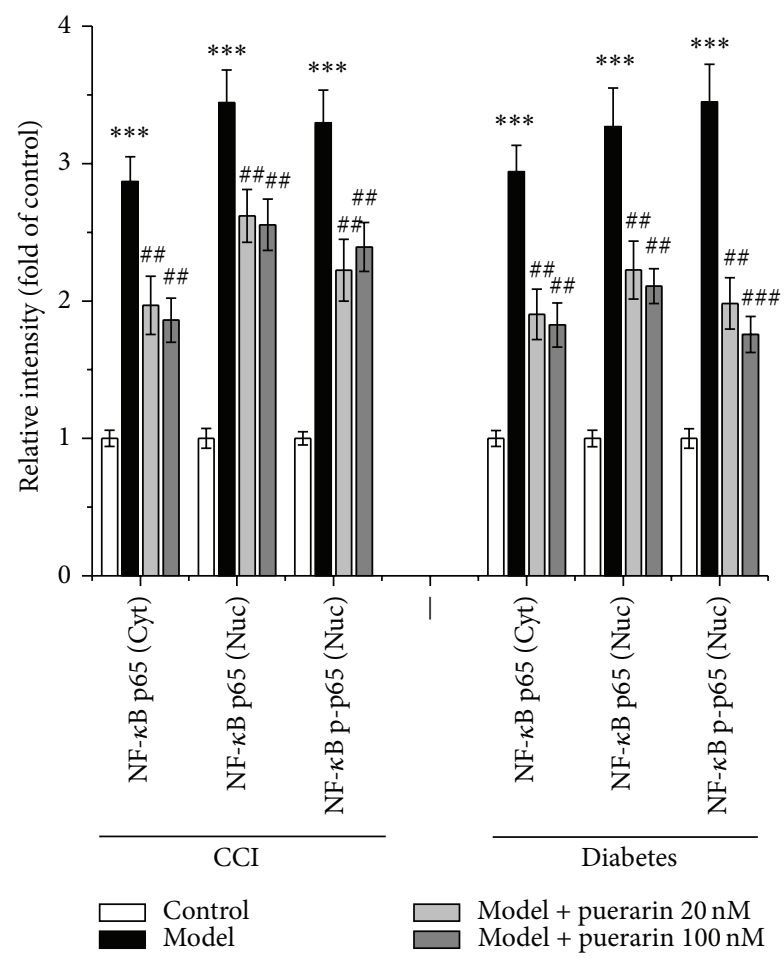

(d)

FIGURE 5: The effects of puerarin on CCI- and diabetes-induced proinflammatory cytokines overexpression (a) and NF- $\kappa \mathrm{B}$ overactivation ((b), (c), (d)) on the 11th day after CCI surgery, and on the 27th day after streptozotocin injection in rat spinal cord. Data are presented as mean \pm SEM. CCI: chronic constriction injury; Cyt: cytoplasmic; Nuc: nuclear. ${ }^{* * *} P<0.001$, CCI and diabetes models versus control; ${ }^{\#} P<0.05,{ }^{\#} P<0.01,{ }^{\# \#} P<0.001$, puerarin versus CCI and diabetes models (one-way ANOVA with post hoc LSD $t$-test). Each group consisted of $6-10$ rats.

can activate NF- $\kappa \mathrm{B}$ or can be activated by NF- $\kappa \mathrm{B}$ [22]. Inhibition of the expression of NF- $\kappa \mathrm{B}$ and proinflammatory factors (TNF- $\alpha$, IL-1 $\beta$, and IL-6) can alleviate mechanical allodynia and thermal hyperalgesia in chronic constriction injury (CCI) rat model [23]. Taken together, NF- $\kappa \mathrm{B}$ is one of the most important transcription factors regulating gene expression of the proinflammatory cytokines $[24,25]$ and is implicated in the initiation and development of neuropathic pain via a neuron-mediated way of central sensitization and glia cells-mediated expressions of proinflammatory cytokines and pain mediators $[6,23,26]$.

Resident astroglia and microglia in the CNS are known to play important roles in neuroinflammation [27]. Spinal glial activation has been demonstrated in a variety of animal pain 
models, including models of neuropathic and inflammatory pain [28]. Following stimulation, spinal glia cells proliferate, undergo morphological changes, increase the expression of cell surface receptors, and increase the production and release of proinflammatory cytokines (TNF- $\alpha$, IL- $1 \beta$, and IL-6) and other cytotoxic products, which in turn enhance pain transmission in the dorsal horn of the spinal cord [28, 29]. Also, the spinal neuroimmune and neuroinflammatory activation is a positive feed-forward loop mediated by proinflammatory cytokines, glia cells, and NF- $\kappa \mathrm{B}$ [28].

In the current study, two classic models for neuropathic pain: a sciatic nerve chronic constriction injury model [30] and a diabetic neuropathy model $[31,32]$, were used. We showed that fluorocitrate, which specifically blocks glial metabolic activity by inhibiting the activity of aconitase, a Krebs cycle enzyme found exclusively in glia [33-36] and served as positive control in this study, reduced the pain response in both models as previous reported [12, 37, 38]. Consistent with the previous studies [5, 23, 27, 39-42], the expression of TNF- $\alpha$, IL-1 $\beta$ IL- 6 , and NF- $\kappa$ B p65 (p-p65) was dramatically increased in spinal cord, and the spinal astroglia and microglia were drastically activated in both the CCI injury and diabetic models. The effects of puerarin were dose-dependent and comparable to that of fluorocitrate. Puerarin did not affect the pain threshold in normal rats, suggesting the effects of puerarin on neuropathic pain are specific. The fact that puerarin inhibited the overexpression of spinal TNF- $\alpha$, IL- $1 \beta$, IL-6, and NF- $\kappa$ B p 65 (p-p65) and spinal glia activation associated with neuropathic pain in our study suggest that the action of puerarin against neuropathic pain involves the regulation of the neuroinflammatory process and the neuroimmune system in general (Figure 6).

\section{Conclusion}

In summary, intrathecal administration of puerarin produces dose-dependent antinociceptive effects in CCI- and diabetesinduced neuropathic pain. One possible mechanism is the inhibition of neuroinflammatory process and glia activation. The present study also suggests that puerarin is a promising platform for developing novel agents for the treatment of neuropathic pain.

\section{Conflict of Interests}

The authors declare that there is no conflict of interests regarding the publication of this paper.

\section{Acknowledgments}

This work was supported by grants from National Natural Science Foundation to Shuyu Yang (30973912), Suhuan Liu (81270901), and Xuejun Li (81073113); Key Project of Fujian Provincial Science and Technology Planning programs (2012D60) and Xiamen Innovation Program for Outstanding Youth Scientist (2011S0446) to Suhuan Liu; and Xiamen Science and Technology Bureau (Xiamen Research Platform for Systems Biology of Metabolic Disease, 3502Z20100001).

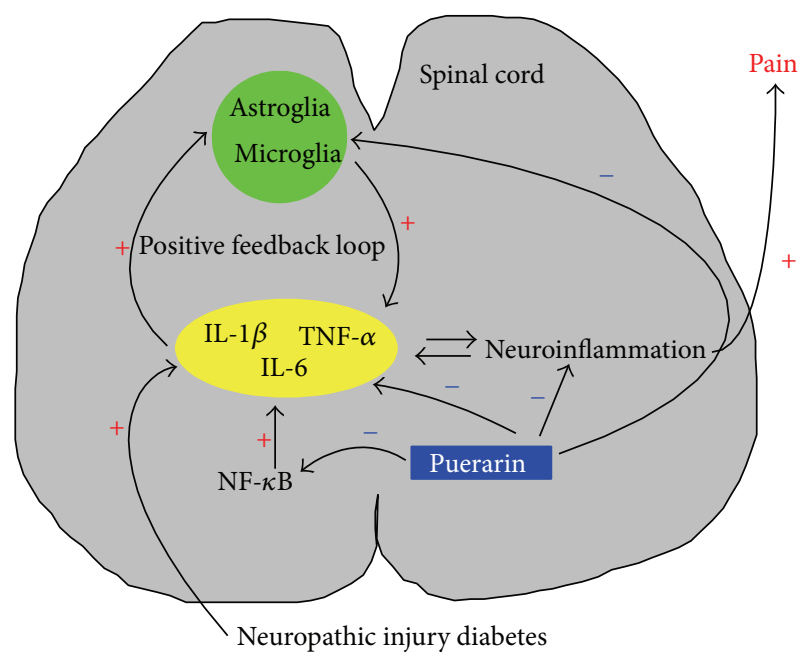

FIGURE 6: Hypothetical mechanisms of antineuroinflammation activity of puerarin.

\section{References}

[1] J. N. Campbell and R. A. Meyer, "Mechanisms of neuropathic pain," Neuron, vol. 52, no. 1, pp. 77-92, 2006.

[2] G. Varrassi, G. Müller-Schwefe, J. Pergolizzi et al., "Pharmacological treatment of chronic pain the need for CHANGE," Current Medical Research and Opinion, vol. 26, no. 5, pp. 12311245, 2010.

[3] P. J. Austin and G. Moalem-Taylor, "The neuro-immune balance in neuropathic pain: involvement of inflammatory immune cells, immune-like glial cells and cytokines," Journal of Neuroimmunology, vol. 229, no. 1-2, pp. 26-50, 2010.

[4] R. Vallejo, D. M. Tilley, L. Vogel, and R. Benyamin, "The role of glia and the immune system in the development and maintenance of neuropathic pain," Pain Practice, vol. 10, no. 3, pp. 167-184, 2010.

[5] Y. Kawasaki, L. Zhang, J. K. Cheng, and R. Ji, “Cytokine mechanisms of central sensitization: Distinct and overlapping role of interleukin-1 $\beta$, interleukin-6, and tumor necrosis factor- $\alpha$ in regulating synaptic and neuronal activity in the superficial spinal cord," The Journal of Neuroscience, vol. 28, no. 20, pp. 5189-5194, 2008.

[6] L. R. Watkins and S. F. Maier, "Glia: a novel drug discovery target for clinical pain," Nature Reviews Drug Discovery, vol. 2, no. 12, pp. 973-985, 2003.

[7] Z. Zhang, T. N. Lam, and Z. Zuo, "Radix Puerariae: an overview of its chemistry, pharmacology, pharmacokinetics, and clinical use," Journal of Clinical Pharmacology, vol. 53, no. 8, pp. 787-811, 2013.

[8] G. Zheng, C. Yu, and Z. Yang, "Puerarin suppresses production of nitric oxide and inducible nitric oxide synthase in lipopolysaccharide-induced $\mathrm{N} 9$ microglial cells through regulating MAPK phosphorylation, O-GlcNAcylation and NF- $\kappa$ B translocation," International Journal of Oncology, vol. 40, no. 5, pp. 1610-1618, 2012.

[9] A. K. Singh, Y. Jiang, S. Gupta, M. Younus, and M. Ramzan, "Anti-inflammatory potency of nano-formulated puerarin and curcumin in rats subjected to the lipopolysaccharide-induced inflammation," Journal of Medicinal Food, vol. 16, no. 10, pp. 899-911, 2013. 
[10] Y. Liu, L. L. Shao, and W. Pang, "Induction of adhesion molecule expression in co-culture of human bronchial epithelial cells and neutrophils suppressed by puerarin via down-regulating p38 mitogen-activated protein kinase and nuclear factor kappa B pathways," Chinese Journal of Integrative Medicine, vol. 20, no. 5, pp. 360-368, 2014.

[11] R. M. LoPachin, T. A. Rudy, and T. L. Yaksh, "An improved method for chronic catheterization of the rat spinal subarachnoid space," Physiology and Behavior, vol. 27, no. 3, pp. 559-561, 1981.

[12] S. Talbot, E. Chahmi, J. P. Dias, and R. Couture, "Key role for spinal dorsal horn microglial kinin $\mathrm{B}_{1}$ receptor in early diabetic pain neuropathy," Journal of Neuroinflammation, vol. 7, article 36, 2010.

[13] G. J. Bennett and Y. K. Xie, "A peripheral mononeuropathy in rat that produces disorders of pain sensation like those seen in man," Pain, vol. 33, no. 1, pp. 87-107, 1988.

[14] S. Mitrirattanakul, N. Ramakul, A. V. Guerrero et al., "Sitespecific increases in peripheral cannabinoid receptors and their endogenous ligands in a model of neuropathic pain," Pain, vol. 126, no. 1-3, pp. 102-114, 2006.

[15] I. E. Tchivileva, A. G. Nackley, L. Qian, S. Wentworth, M. Conrad, and L. B. Diatchenko, "Characterization of NF- $\kappa$ Bmediated inhibition of catechol-O-methyltransferase," Molecular Pain, vol. 5, article 13, 2009.

[16] J. Cheng, R. C. Chou, L. Hwang, and L. Chiou, "Antiallodynic effects of intrathecal orexins in a rat model of postoperative pain," Journal of Pharmacology and Experimental Therapeutics, vol. 307, no. 3, pp. 1065-1071, 2003.

[17] J. A. DeLeo and R. P. Yezierski, "The role of neuroinflammation and neuroimmune activation in persistent pain," Pain, vol. 90, no. 1-2, pp. 1-6, 2001.

[18] H. Hashizume, J. A. DeLeo, R. W. Colburn, and J. N. Weinstein, "Spinal glial activation and cytokine expression after lumbar root injury in the rat," Spine, vol. 25, no. 10, pp. 1206-1217, 2000.

[19] K. Okamoto, D. P. Martin, J. D. Schmelzer, Y. Mitsui, and P. A. Low, "Pro- and anti-inflammatory cytokine gene expression in rat sciatic nerve chronic constriction injury model of neuropathic pain," Experimental Neurology, vol. 169, no. 2, pp. 386391, 2001.

[20] G. Moalem and D. J. Tracey, "Immune and inflammatory mechanisms in neuropathic pain," Brain Research Reviews, vol. 51, no. 2, pp. 240-264, 2006.

[21] S. S. Makarov, "NF- $\kappa$ b as a therapeutic target in chronic inflammation: recent advances," Molecular Medicine Today, vol. 6, no. 11, pp. 441-448, 2000.

[22] F. Marchand, M. Perretti, and S. B. McMahon, "Role of the immune system in chronic pain," Nature Reviews Neuroscience, vol. 6, no. 7, pp. 521-532, 2005.

[23] G. Sakaue, M. Shimaoka, T. Fukuoka et al., "NF- $\kappa$ B decoy suppresses cytokine expression and thermal hyperalgesia in a rat neuropathic pain model," NeuroReport, vol. 12, no. 10, pp. 2079-2084, 2001.

[24] T. Hanada and A. Yoshimura, "Regulation of cytokine signaling and inflammation," Cytokine and Growth Factor Reviews, vol. 13, no. 4-5, pp. 413-421, 2002.

[25] H. Li and X. Lin, "Positive and negative signaling components involved in TNF $\alpha$-induced NF- $\kappa \mathrm{B}$ activation," Cytokine, vol. 41, no. 1, pp. 1-8, 2008.

[26] W. Ma and M. A. Bisby, "Increased activation of nuclear factor kappa B in rat lumbar dorsal root ganglion neurons following partial sciatic nerve injuries," Brain Research, vol. 797, no. 2, pp. 243-254, 1998.

[27] E. D. Milligan and L. R. Watkins, "Pathological and protective roles of glia in chronic pain," Nature Reviews Neuroscience, vol. 10, no. 1, pp. 23-36, 2009.

[28] E. A. Old and M. Malcangio, "Chemokine mediated neuron-glia communication and aberrant signalling in neuropathic pain states," Current Opinion in Pharmacology, vol. 12, no. 1, pp. 6773, 2012.

[29] M. R. Suter, Y. R. Wen, I. Decosterd, and R. Ji, "Do glial cells control pain?" Neuron Glia Biology, vol. 3, no. 3, pp. 255-268, 2007.

[30] N. Attal and D. Bouhassira, "Neuropathic pain: experimental advances and clinical applications," Revue Neurologique, vol. 160, no. 2, pp. 199-203, 2004.

[31] N. A. Calcutt, "Potential mechanisms of neuropathic pain in diabetes," International Review of Neurobiology, vol. 50, pp. 205$228,2002$.

[32] Z. Simmons and E. L. Feldman, "Update on diabetic neuropathy," Current Opinion in Neurology, vol. 15, no. 5, pp. 595-603, 2002.

[33] B. Hassel, R. E. Paulsen, A. Johnsen, and F. Fonnum, "Selective inhibition of glial cell metabolism in vivo by fluorocitrate," Brain Research, vol. 576, no. 1, pp. 120-124, 1992.

[34] R. E. Paulsen, A. Contestabile, L. Villani, and F. Fonnum, "An in vivo model for studying function of brain tissue temporarily devoid of glial cell metabolism: the use of fluorocitrate," Journal of Neurochemistry, vol. 48, no. 5, pp. 1377-1385, 1987.

[35] E. D. Milligan, C. Twining, M. Chacur et al., "Spinal glia and proinflammatory cytokines mediate mirror-image neuropathic pain in rats," The Journal of Neuroscience, vol. 23, no. 3, pp. 10261040, 2003.

[36] T. Nakagawa, K. Wakamatsu, N. Zhang et al., "Intrathecal administration of ATP produces long-lasting allodynia in rats: differential mechanisms in the phase of the induction and maintenance," Neuroscience, vol. 147, no. 2, pp. 445-455, 2007.

[37] H. Obata, J. C. Eisenach, H. Hussain, T. Bynum, and M. Vincler, "Spinal glial activation contributes to postoperative mechanical hypersensitivity in the rat," The Journal of Pain, vol. 7, no. 11, pp. 816-822, 2006.

[38] J. Mika, "Modulation of microglia can attenuate neuropathic pain symptoms and enhance morphine effectiveness," Pharmacological Reports, vol. 60, no. 3, pp. 297-307, 2008.

[39] Y. P. Zhang, C. Y. Song, Y. Yuan et al., "Diabetic neuropathic pain development in type 2 diabetic mouse model and the prophylactic and therapeutic effects of coenzyme Q10," Neurobiology of Disease, vol. 58, pp. 169-178, 2013.

[40] Y. W. Chen, P. L. Hsieh, Y. C. Hung, and J. T. Cheng, "Physical exercise induces excess hsp72 expression and delays the development of hyperalgesia and allodynia in painful diabetic neuropathy rats," Anesthesia and Analgesia, vol. 116, no. 2, pp. 482-490, 2013.

[41] P. Ren, Y. Zhang, X. Zhang et al., "High-mobility group box 1 contributes to mechanical allodynia and spinal astrocytic activation in a mouse model of type 2 diabetes," Brain Research Bulletin, vol. 88, no. 4, pp. 332-337, 2012.

[42] Y. R. Wen, P. H. Tan, J. K. Cheng, Y. Liu, and R. Ji, "Microglia: a promising target for treating neuropathic and postoperative pain, and morphine tolerance," Journal of the Formosan Medical Association, vol. 110, no. 8, pp. 487-494, 2011. 


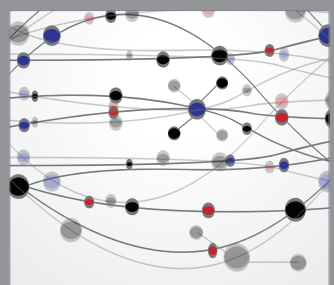

The Scientific World Journal
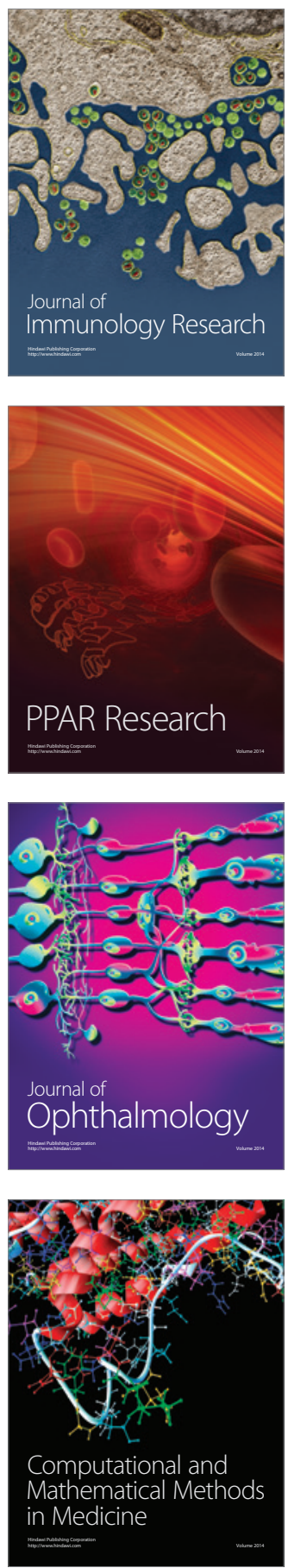

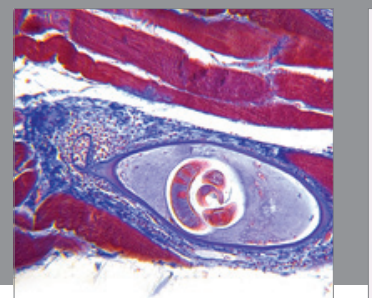

Gastroenterology

Research and Practice
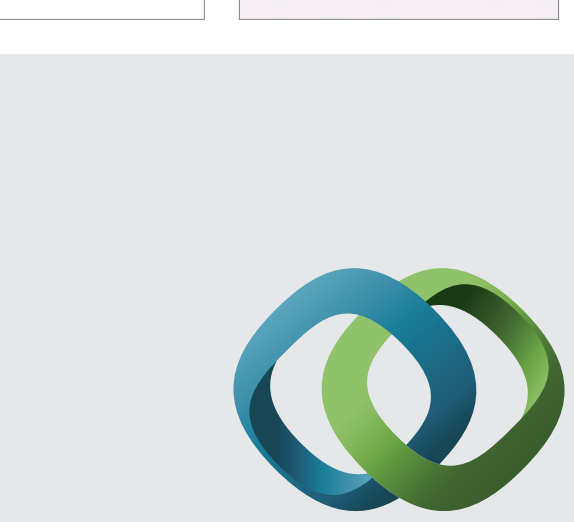

\section{Hindawi}

Submit your manuscripts at

http://www.hindawi.com
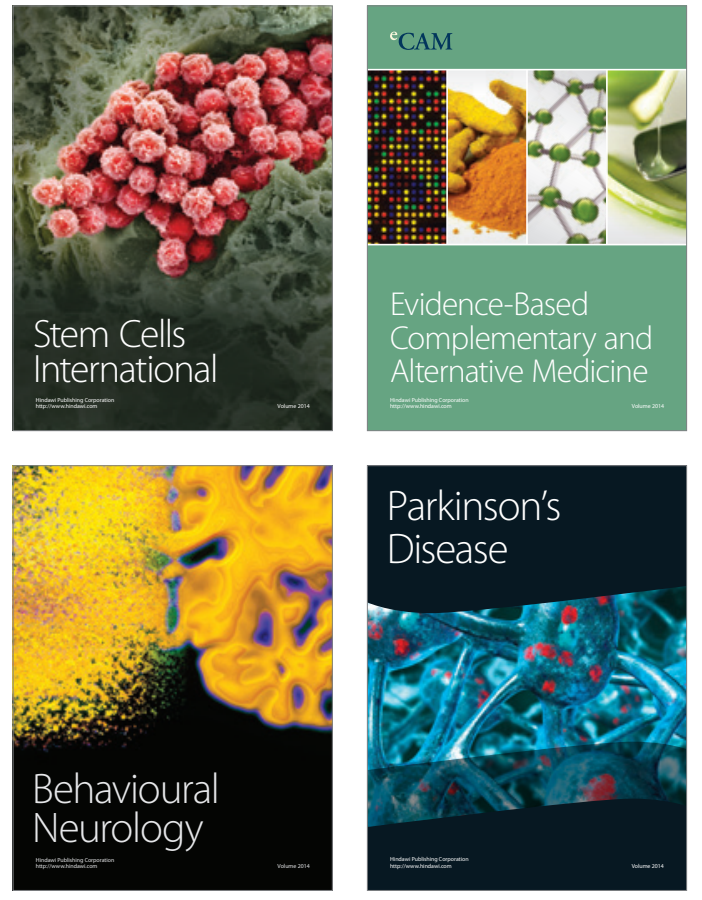
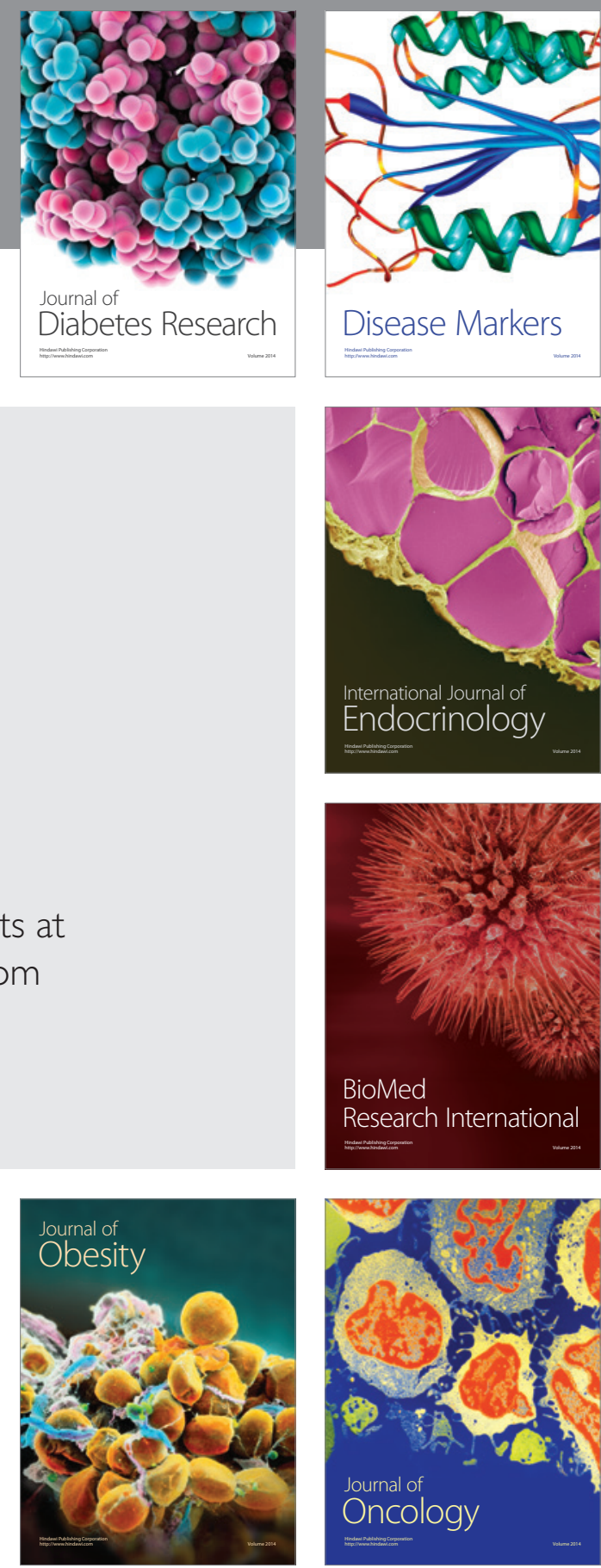

Disease Markers
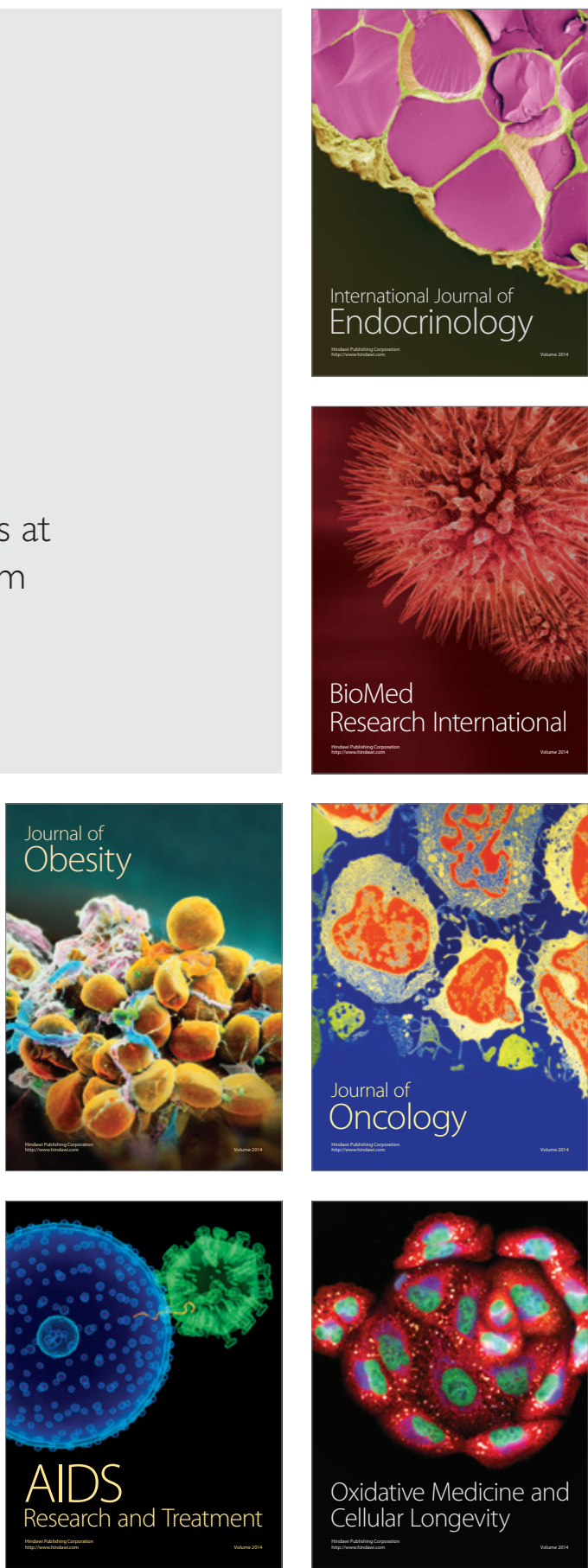